\title{
Hydrogen-containing saline alleviates pressure overload-induced interstitial fibrosis and cardiac dysfunction in rats
}

\author{
JING YANG $^{1}$, SHUJING WU ${ }^{1,2}$, LIQUN ZHU $^{3}$, JINGJING CAI $^{1,4}$ and $\mathrm{LU} \mathrm{FU}^{1}$ \\ ${ }^{1}$ Department of Cardiovascular Medicine, The First Affiliated Hospital of Harbin Medical University, \\ Harbin, Heilongjiang 150001; ${ }^{2}$ Department of Cardiology, The ChengGong Hospital Affiliated to Xiamen University, \\ Xiamen, Fujian 361003; ${ }^{3}$ Department of Emergency Internal Medicine, The First Affiliated Hospital of \\ Harbin Medical University, Harbin, Heilongjiang 150001; ${ }^{4}$ Department of Cardiology, The \\ Xingan League People's Hospital, Xingan, Inner Mongolia 137400, P.R. China
}

Received July 16, 2016; Accepted April 27, 2017

DOI: $10.3892 / \mathrm{mmr} .2017 .6849$

\begin{abstract}
Cardiac fibrosis induced by sustained pressure overload contributes to heart failure. Oxidative stress serves an important role in cardiac remodeling and heart failure independent of etiological factors. The application of hydrogen as an antioxidant is a novel concept in disease treatment, however no studies as present have investigated the effects of hydrogen on cardiac fibrosis. In the present study, the effects of hydrogen on pressure overload-induced cardiac fibrosis and heart failure were investigated in abdominal aortic-constricted rats. Masson's trichrome staining and echocardiography were used to evaluate the fibrotic area and cardiac function, respectively. Reactive oxygen species (ROS) content was detected by immunofluorescence. Malondialdehyde (MDA) concentration, the activity of superoxide dismutase (SOD) and hydroxyproline content were measured by spectrophotometry. Western blot analysis was used to detect the protein levels of transforming growth factor (TGF)- $\beta 1$, connective tissue growth factor (CTGF), NADPH oxidases (NOX)2, NOX4, p38 mitogen-activated protein kinase (MAPK) and Smad2/3. Reverse transcription-quantitative polymerase chain reaction was performed to detect the mRNA expression of collagen I ( Col I) and fibronectin 1 (FN1). Hydrogen-containing saline (HCS) treatment was observed to improve interstitial fibrosis and cardiac function and to decrease the level of ROS, the oxidative-stress marker MDA and expression of NOXs, while increasing the activity of the anti-oxidant enzyme SOD. HCS treatment also
\end{abstract}

Correspondence to: $\mathrm{Dr} \mathrm{Lu} \mathrm{Fu}$, Department of Cardiovascular Medicine, The First Affiliated Hospital of Harbin Medical University, 23 Youzheng Street, Harbin, Heilongjiang 150001, P.R. China

E-mail: fulunadia@163.com

Key words: pressure overload, cardiac fibrosis, hydrogen-containing saline, oxidative stress, NADPH oxidase, transforming growth factor $\beta 1$ decreased the phosphorylation of p38 MAPK and Smad2/3, and the expression of TGF- $\beta 1$ and CTGF, which were accompanied by reduced hydroxyproline content, Col I and FNI mRNA levels. These results indicate that HCS treatment can improve cardiac function by reducing interstitial fibrosis in pressure-overloaded rats through its anti-oxidative properties and via suppression of TGF- $\beta 1$ signaling.

\section{Introduction}

Heart failure is the terminal stage of various cardiovascular diseases and is a global problem with high prevalence (1). Sustained pressure overload associated with hypertension can induce cardiac fibrosis, which is characterized by excess deposition of collagen and other extracellular matrix (ECM) components in the interstitial and peri-vascular regions of the myocardium that lead to myocardial stiffness and ultimately, to heart failure (2). Therapeutic strategies that reduce cardiac fibrosis may therefore block the progression of cardiac fibrosis to heart failure in hypertensive heart diseases.

Transforming growth factor (TGF)- $\beta 1$ serves an essential role in cardiac fibrosis by inducing the proliferation of cardiac fibroblasts and stimulating ECM deposition (3). It has been reported that TGF- $\beta 1$ antagonists can prevent myocardial fibrosis and heart failure (4). Smad2/3 is the important downstream signaling of TGF- $\beta 1$, and connective tissue growth factor (CTGF) also serves as a critical downstream target gene of TGF- $\beta 1$ to prompt fibrosis (5). The generation of reactive oxygen species (ROS) by nicotinamide adenine dinucleotide phosphate oxidases (NOX) is increased under pressure overload, and induces other enzymes such as xanthine oxidase and nitric oxide synthase to enhance ROS formation (6). NOX2 and NOX4 are notably relevant to cardiac pathophysiology. Excessive ROS levels can directly affect matrix protein expression and metabolism (2) in addition to cytokine and growth factor signaling in cardiac fibrosis. For example, the upregulation of TGF- $\beta 1$ by angiotensin (Ang) II is mediated by NOX-induced ROS, which activates downstream ROS-sensitive kinases such as p38 mitogen-activated protein kinase (MAPK) in cardiomyocytes and fibroblasts (7). In addition, enalaprilat, an AngII-converting enzyme inhibitor, 
suppresses cardiac fibroblast proliferation induced by Ang II by blocking NOX/ROS/p38 MAPK/TGF- $\beta 1$ signaling (8).

Hydrogen $\left(\mathrm{H}_{2}\right)$ has been proposed as a medical gas in prophylactic and therapeutic applications with a diverse physiological profile. Due to the fact that $\mathrm{H}_{2}$ was demonstrated to mitigate ischemia/reperfusion (I/R) injury in the brain owing to its anti-oxidative properties (9), a number of studies have reported its biological effects against oxidative stress and other aspects, including anti-inflammatory (10), anti-apoptotic (11), anti-allergenic (12) and metabolism-stimulating effects (13). In the cardiovascular system, $\mathrm{H}_{2}$ treatment protects against myocardial I/R injury $(14,15)$, the development of atherosclerosis (16) and radiation-induced cellular damage (17) while enhancing cardiac graft transplantation (18) and reducing left ventricular (LV) hypertrophy (19). However, the benefits of $\mathrm{H}_{2}$ for pressure overload-induced cardiac fibrosis remain unclear. The present study investigated the effects and underlying mechanisms of hydrogen-containing saline (HCS) treatment in a rat model of interstitial fibrosis and cardiac dysfunction induced by abdominal aortic constriction (AC).

\section{Materials and methods}

Animals and ethics. A total of 60 adult (6-week-old) male Wistar rats (150-200 g) from the Laboratory Animal Center of the First Affiliated Hospital of Harbin Medical University (Harbin, China) were maintained in a temperature- and humidity-controlled room with free access to food and water for 1 week prior to the operation. Experimental procedures were in compliance with the Guide for the Care and Use of Laboratory Animals of the National Research Council of China (20) and were approved by the Ethics Committee of the First Affiliated Hospital of Harbin Medical University.

HCS preparation. HCS was produced by introducing $\mathrm{H}_{2}$ gas (flow rate: $11 / \mathrm{min}$ ) into $500 \mathrm{ml}$ of $0.9 \%$ saline solution with stirring for $10 \mathrm{~min}$ until reaching the point of saturation (21). HCS was freshly prepared every week and stored at $4^{\circ} \mathrm{C}$ under atmospheric pressure in an aluminum bag with no dead volume. Hydrogen content in the saline and in animal blood was confirmed by gas chromatography as previously described (9).

Abdominal AC and HCS administration. Rats were subjected to suprarenal abdominal AC as previously described (22). Briefly, rats were anaesthetized by intraperitoneal injection of $10 \%$ chloral hydrate $(3 \mathrm{ml} / \mathrm{kg})$, and a $4-0$ suture was tied around the suprarenal aorta with a 21-gauge needle placed alongside to yield an internal diameter of $0.8 \mathrm{~mm}$. A total of three days later, surviving $\mathrm{AC}$ rats were randomly assigned to the $\mathrm{AC}+$ saline $(\mathrm{AC} ; \mathrm{n}=7)$ and $\mathrm{AC}+\mathrm{HCS}$ treatment $\left(\mathrm{AC}+\mathrm{H}_{2}\right.$; $\mathrm{n}=7$ ) groups for 16 weeks, during which time $10 \mathrm{ml} / \mathrm{kg}$ of HCS or saline was administered every morning to the animals via intraperitoneal injection. Randomly selected normal rats served as the control group (Con; $n=8)$, and were administrated with the same dose of saline.

Echocardiography. Transthoracic echocardiography was performed prior and subsequent to HCS administration with an ultrasound apparatus (SONOS 7500; Philips, Rotterdam,
The Netherlands) equipped with a 12-MHz transducer. LV end-diastolic diameter (LVDd), LV end-systolic diameter (LVSd), interventricular septum thickness (IVSd) and LV posterior wall thickness (LVPWd) were measured. LV ejection fraction (EF) and fractional shortening (FS) were calculated from M-mode recordings. Measurements from three consecutive cardiac cycles were averaged and data were analyzed by a blinded observer.

Morphometric and histopathological analysis. Following echocardiographic measurements and blood sample collection, the heart was dissected and weighed. The LV was separated from the atria, right ventricle and the large vessels then was weighed, immersion-fixed in $4 \%$ buffered paraformaldehyde and flash frozen in liquid nitrogen until use. Heart weight (HW) and LV weight (LVW) were used to calculate the $\mathrm{HW} /$ body weight $(\mathrm{BW})$ and $\mathrm{LVW} / \mathrm{BW}$ ratios. Paraffin-embedded tissue was cut into $4 \mu \mathrm{m}$ serial sections that were stained with Masson's trichrome to evaluate ECM deposition. Digital images of 10 fields were randomly selected from each section of the LV by a light microscope (Olympus Corporation, Tokyo, Japan) and the percent of fibrosis area was analyzed with Image-Pro Plus 6.0 image analysis software (Media Cybernetics, Inc., Rockville, MD, USA).

Measurement of ROS. Intracellular ROS production in the LV tissues was detected by the use of 2,7-dichlorofluorescin diacetate (CM-H2DCFDA) (Shanghai Genmed, Gene Pharmaceutical Technology Co., Ltd., Shanghai, China) fluorescent dye. Briefly, frozen heart tissues were incubated with $500 \mu 1$ CM-H2DCFDA and dissolved in Reagent C (500:1, Shanghai Genmed, Gene Pharmaceutical Technology Co., Ltd.) at $37^{\circ} \mathrm{C}$ for $20 \mathrm{~min}$. Fluorescence was detected by confocal laser scanning microscopy with red light and measuring the intensity of red fluorescence, where the ROS content increased in proportion to the intensity of red fluorescence.

Measurement of malondialdehyde (MDA) concentration and superoxide dismutase (SOD) activity and hydroxyproline content. MDA is a marker of oxidant-mediated lipid peroxidation, whereas SOD is an antioxidant enzyme. Hydroxyproline, the decomposition product of collagen, is considered to be an index of collagen quantity. MDA concentration, SOD activity and hydroxyproline content were measured in LV tissue homogenates using commercial kits (Nanjing Jiancheng Bioengineering Research Institute, Nanjing, China) according to the manufacturer's protocols. For the MDA and SOD assay, frozen tissues were homogenized in a saline buffer and centrifuged at $1,082 \mathrm{x}$ g for $10 \mathrm{~min}$ at room temperature to obtain supernatant, and then were determined its protein concentration. For detecting hydroxyproline, frozen tissues were weighed, hydrolyzed with alkali and centrifuged at 2,121 x g for $10 \mathrm{~min}$ at room temperature to obtain the supernatant. MDA concentration, SOD activity and hydroxyproline content were measured by spectrophotometer colorimetry (722G; Shanghai Jingke Scientific Instrument Co., Ltd., Shanghai, China) at 532, 450 and $550 \mathrm{~nm}$, respectively. The final results of MDA concentration and SOD activity were corrected for protein content, and hydroxyproline content was expressed as $\mu \mathrm{g} / \mathrm{g}$ wet weight. 
Western blot analysis. Frozen LV tissue was mechanically lysed using RIPA buffer (Beyotime Institute of Biotechnology, Shanghai, China), then centrifuged at $12,000 \times \mathrm{g}$ and $4^{\circ} \mathrm{C}$ for $10 \mathrm{~min}$. Protein concentration was estimated with the Bicinchoninic Acid Protein Assay kit (Beyotime Insitute of Biotechnology). Protein samples (60 $\mu \mathrm{g}$ per lane) were separated by sodium dodecyl sulfate-polyacrylamide gel electrophoresis and transferred to a polyvinylidene difluoride membrane (EMD Millipore, Billerica, MA, USA), which was blocked with $5 \%$ skimmed milk for $2 \mathrm{~h}$ at room temperature and then probed overnight at $4^{\circ} \mathrm{C}$ with rabbit polyclonal antibodies against TGF- $\beta 1$ (1:1,000; GTX110630; GeneTex, Irvine, CA, USA) and NOX4 (1:500, sc-30141, Santa Cruz Biotechnology, Inc., Dallas, TX, USA), rabbit monoclonal antibodies against NOX2 (1:1,000; ab129068; Abcam, Cambridge, MA, USA), phosphorylated-Smad2/3 (p-Smad2/3; 1:1,000; no. 8828; Cell Signaling Technology, Inc., Danvers, MA, USA), Smad2/3 (1:1,000; no. 8685; Cell Signaling Technology, Inc.), p38 MAPK (D13E1) (1:1,000; no. 8690; Cell Signaling Technology, Inc.), p-p38 MAPK (Thr180/Tyr182) (D3F9) (1:1,000; no. 4511; Cell Signaling Technology, Inc.) and mouse monoclonal antibodies against CTGF (E-5) (1:50; sc-365970; Santa Cruz Biotechnology, Inc.) and glyceraldehyde 3-phosphate dehydrogenase (GAPDH; 1:5,000; KC-5G4; KangChen Bio-tech, Inc., Shanghai, China). The membrane was washed three times for $10 \mathrm{~min}$ with phosphate-buffered saline containing $0.5 \%$ Tween-20, then incubated for $2 \mathrm{~h}$ with the secondary goat antibodies against rabbit (ZDR-5306) or mouse (ZDR-5307) IgG conjugated to horseradish peroxidase (1:2,000; OriGene Technologies, Beijing, China). Protein bands were visualized by enhanced chemiluminescence using western blot detection reagents (Thermo Fisher Scientific, Inc., Waltham, MA, USA) and exposed to X-ray film, which was digitized with a scanner (LiED 110; Canon, Inc., Tokyo, Japan). The band intensity (area $\mathrm{x}$ optical density) in each group was measured using ImageJ $1.45 \mathrm{~s}$ software (National Institutes of Health, Bethesda, MD, USA) and normalized to that of GAPDH.

Reverse transcription-quantitative polymerase chain reaction (RT-qPCR). Total RNA was extracted from LV tissue samples using RNAiso Plus (Takara Bio, Inc., Otsu, Japan) and $1 \mu \mathrm{g}$ was reverse transcribed into cDNA, using the PrimeScript RT Reagent kit with gDNA Eraser (Takara Bio, Inc.) and M-MLV Reverse Transcriptase (Takara Biotechnology Co., Ltd., Dalian, China), according to the manufacturer's protocol. RT-qPCR analysis was conducted with SYBR-Green (Roche Diagnostics GmbH, Mannheim, Germany) on an ABI 7500 RT-PCR system (Applied Biosystems; Thermo Fisher Scientific, Inc.) in order to determine the mRNA levels of collagen I (Col I), fibronectin 1 (FNI) and GAPDH. The primer sequences were as follows: Col I, 5'-GACTGGCAACCTCAA GAAGG-3' (forward) and 5'-GACTGTCTTGCCCCAAGT TC-3' (reverse); FN1, 5'-TACACGGTTTCCCATTACGC-3' (forward) and 5'-CCTTTCCATTCCCGAGACAT-3' (reverse); GAPDH, 5'-GGAAAGCTGTGGCGTGAT-3' (forward) and 5'-AAGGTGGAAGAATGGGAGTT-3' (reverse). Among them, GAPDH was used as an internal standard. The qPCR reaction mixture consisted of $10 \mu \mathrm{l}$ SYBR-Green, $0.2 \mu \mathrm{l}$ forward primer, $0.2 \mu \mathrm{l}$ reverse primer, $2 \mu \mathrm{l} \mathrm{cDNA}$ and $7.6 \mu \mathrm{l}$ sterile water. Following the thermal treatment of $15 \mathrm{~min}$ at $95^{\circ} \mathrm{C}$, the reactions were conducted using 45 cycles of $15 \mathrm{sec}$ at $95^{\circ} \mathrm{C}, 30 \mathrm{sec}$ at $55^{\circ} \mathrm{C}, 30 \mathrm{sec}$ at $72^{\circ} \mathrm{C}$, followed by a final extension for $7 \mathrm{~min}$ at $72^{\circ} \mathrm{C}$. The expression of target genes was quantified relative to the level of GAPDH using the $\Delta \Delta \mathrm{Cq}$ method (23).

Statistical analysis. Data are expressed as the mean \pm standard deviation. One-way analysis of variance followed by Tukey's post hoc test was conducted to determine differences among groups using SPSS software, version 20.0 (IBM Corp., Armonk, NY, USA). P $<0.05$ was considered to indicate a statistically significant difference.

\section{Results}

$\mathrm{H}_{2}$ treatment improves cardiac function induced by pressure overload. The effect of $\mathrm{H}_{2}$ on cardiac function was assessed by echocardiography (Table I). Chronic pressure overload resulted in a decline in EF and FS in the AC as compared with the Con group (all $\mathrm{P}<0.001$ ), indicating impaired cardiac function. Treatment with HCS improved both parameters as compared with the AC group (all $\mathrm{P}<0.001)$. Chronic pressure overload also caused the dilation of the heart, as evidenced by increases in LVDd and LVSd in the AC relative to the Con group (all $\mathrm{P}<0.001)$. HCS treatment attenuated the increase in $\mathrm{LV}$ dimension (all $\mathrm{P}<0.001)$. However, there were no differences in IVSd and LVPWd across groups (all $\mathrm{P}>0.05$ ).

In addition, the morphometric parameters of the heart were also examined (Table I). BW was similar across groups. $\mathrm{LVW}, \mathrm{LVW} / \mathrm{BW}$ and $\mathrm{HW} / \mathrm{BW}$ were increased in the $\mathrm{AC}$ as compared with the Con group (all $\mathrm{P}<0.01$ ). However, HCS treatment didn't suppress the increase in LVW, LVW/BW and $\mathrm{HW} / \mathrm{BW}$ as compared with the AC group (all $\mathrm{P}>0.05$ ). These results indicate that HCS treatment improved cardiac function however had no effects on cardiac hypertrophy induced by sustained pressure overload.

$\mathrm{H}_{2}$ treatment reduces interstitial fibrosis induced by pressure overload. ECM deposition was assessed by Masson's trichrome staining (Fig. 1A and B). The interstitial fibrotic area was greater in the $\mathrm{AC}$ as compared with the Con group $(\mathrm{P}<0.001)$. Treatment with HCS reduced ECM deposition $\left(\mathrm{AC}+\mathrm{H}_{2}\right.$ vs. $\left.\mathrm{AC} ; \mathrm{P}<0.001\right)$. Consistent with the above results, the LV hydroxyproline content (Fig. 1C) was increased in the $\mathrm{AC}$ as compared with the Con group $(\mathrm{P}<0.001)$, whereas the content was decreased in $\mathrm{HCS}$-treated rats $\left(\mathrm{AC}+\mathrm{H}_{2}\right.$ vs. $\mathrm{AC}$; $\mathrm{P}<0.01)$. In the AC group, the mRNA levels of Col I and FNI, two ECM components, were upregulated by approximately 2-fold and 6-fold, respectively, as compared with control rats (all $\mathrm{P}<0.001$; Fig. 1D and E); HCS treatment inhibited the expression of both transcripts to $68 \%$ for $\mathrm{Col} I$ and $65 \%$ for FN1 of the levels in the AC group (all $\mathrm{P}<0.01$ ).

$\mathrm{H}_{2}$ abolishes the increase in TGF- $\beta 1$ and its downstream genes induced by pressure overload. TGF- $\beta 1$ serves a key role in the development of cardiac fibrosis. AC resulted in an increase in $T G F-\beta 1$ protein level compared with control rats $(\mathrm{P}<0.001$; Fig. 2A and B). HCS treatment abolished the AC-induced upregulation of TGF- $\beta 1(\mathrm{P}<0.001)$. The phosphorylation of 
Table I. Effects of $\mathrm{H}_{2}$ on echocardiographic and morphometric parameters.

\begin{tabular}{lccc}
\hline Parameter & Con $(\mathrm{n}=8)$ & $\mathrm{AC}(\mathrm{n}=7)$ & $\mathrm{AC}+\mathrm{H}_{2}(\mathrm{n}=7)$ \\
\hline IVSd $(\mathrm{mm})$ & $1.64 \pm 0.07$ & $1.76 \pm 0.10$ & $1.72 \pm 0.09$ \\
LVPWd (mm) & $1.63 \pm 0.07$ & $1.71 \pm 0.08$ & $1.67 \pm 0.06$ \\
BW $(\mathrm{g})$ & $381.86 \pm 15.03$ & $390.29 \pm 13.87$ & $375.29 \pm 10.81$ \\
LVW $(\mathrm{g})$ & $0.85 \pm 0.08$ & $1.02 \pm 0.07^{\mathrm{a}}$ & $0.94 \pm 0.06$ \\
LVW/BW (mg/g) & $2.23 \pm 0.12$ & $2.60 \pm 0.09^{\mathrm{b}}$ & $2.49 \pm 0.10$ \\
HW/BW (mg/g) & $2.88 \pm 0.12$ & $3.34 \pm 0.13^{\mathrm{b}}$ & $3.23 \pm 0.10$ \\
LVDd (mm) & $6.00 \pm 0.17$ & $7.05 \pm 0.19^{\mathrm{b}}$ & $6.52 \pm 0.12^{\mathrm{c}}$ \\
LVSd (mm) & $3.51 \pm 0.15$ & $5.63 \pm 0.24^{\mathrm{b}}$ & $4.82 \pm 0.09^{\mathrm{c}}$ \\
FS $(\%)$ & $41.56 \pm 1.79$ & $20.11 \pm 1.44^{\mathrm{b}}$ & $26.13 \pm 1.04^{\mathrm{c}}$ \\
EF $(\%)$ & $79.99 \pm 1.86$ & $48.96 \pm 2.77^{\mathrm{b}}$ & $59.67 \pm 1.69^{\mathrm{c}}$
\end{tabular}

Data are presented as the mean \pm standard deviation. ${ }^{\mathrm{a}} \mathrm{P}<0.01,{ }^{\mathrm{b}} \mathrm{P}<0.001 \mathrm{vs}$. control group; ${ }^{\mathrm{c}} \mathrm{P}<0.001$ vs. AC group. Con, control; AC, aortic constriction; IVSd, interventricular septum thickness; LVPWd, left ventricle posterior wall thickness; BW, body weight; LVW, left ventricular weight; HW, heart weight; LVDd, left ventricular end-diastolic diameter; LVSd, left ventricular end-systolic diameter; FS, fractional shortening; EF, ejection fraction.

A

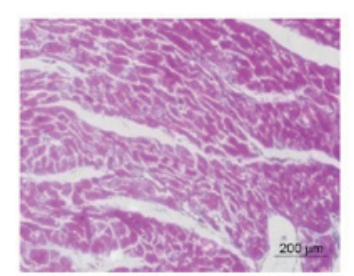

Con

B
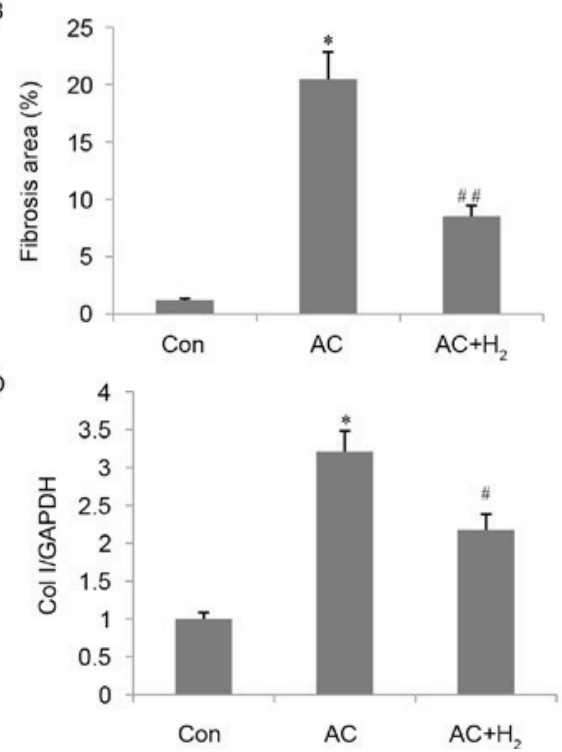

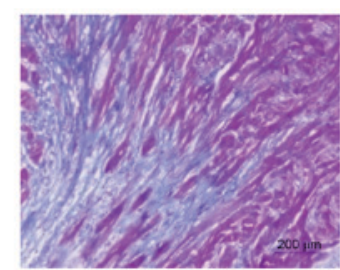

AC

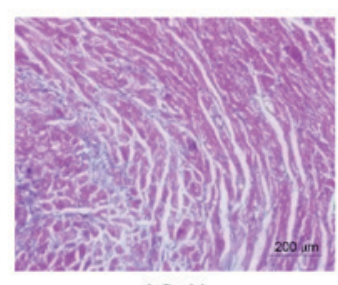

$\mathrm{AC}+\mathrm{H}_{2}$

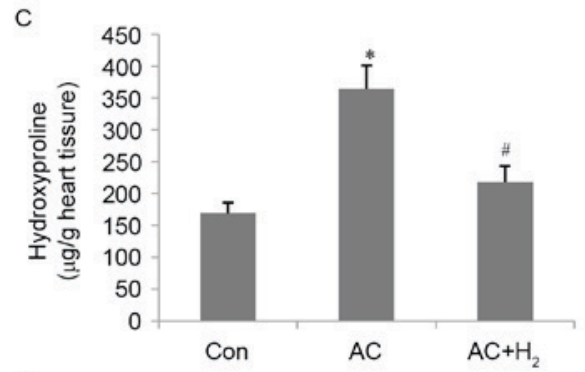

E

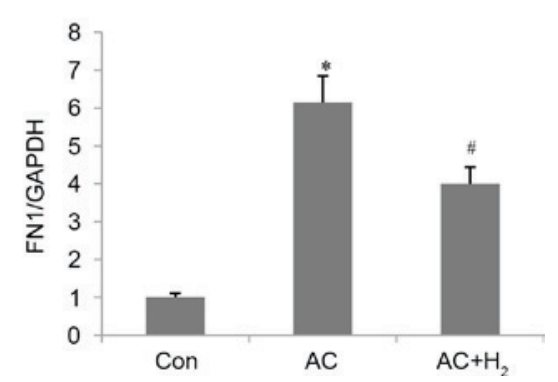

Figure 1. Effect of $\mathrm{H}_{2}$ on cardiac interstitial fibrosis in pressure-overloaded hearts. (A) Representative image (magnification, x200) of heart sections stained by Masson's trichrome. (B) Quantitative analysis of fibrotic area. (C) Hydroxyproline content in the LV. mRNA expression level of (D) Col I and (E) FNI in the LV. Data are presented as the mean \pm standard deviation ( $\mathrm{n}=4$ for $\mathrm{B}, \mathrm{n}=3$ for $\mathrm{C}, \mathrm{D}, \mathrm{E})$. ${ }^{*} \mathrm{P}<0.001$ vs. Con group; ${ }^{\sharp} \mathrm{P}<0.01$, ${ }^{\# \prime} \mathrm{P}<0.001$ vs. AC group. LV, left ventricle; Col I, collagen I; FN1, fibronectin 1; Con, control; AC, aortic constriction.

Smad2/3 and CTGF protein level were upregulated following the tendency of TGF- $\beta 1$ in AC group when compared with the Con group (all $\mathrm{P}<0.01$; Fig. $2 \mathrm{~A}, \mathrm{C}$ and $\mathrm{D}$ ), however significantly decreased in the $\mathrm{AC}+\mathrm{H}_{2}$ group $\left(\mathrm{AC}+\mathrm{H}_{2}\right.$ vs. $\left.\mathrm{AC} ; \mathrm{P}<0.05\right)$. These results indicate that hydrogen inhibits TGF- $\beta 1$ and its downstream signaling.
$\mathrm{H}_{2}$ reduced $\mathrm{ROS}$ formation in the myocardium. ROS production in the LV tissues was detected by CM-H2DCFDA fluorescence (Fig. 3). The ROS content, indicating a higher concentration of red fluorescent particles, was significantly increased in the AC group, compared with the Con group. However, HCS treatment decreased the ROS level of the AC group. 
A

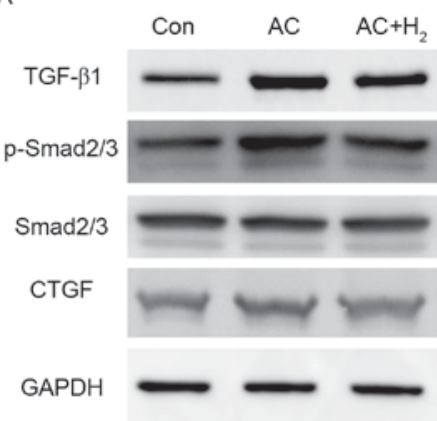

C

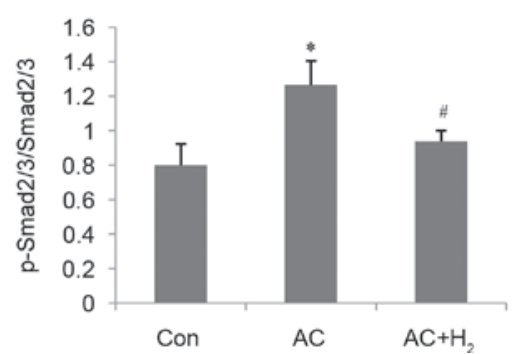

B

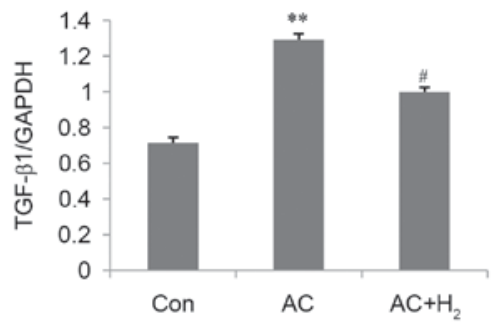

D

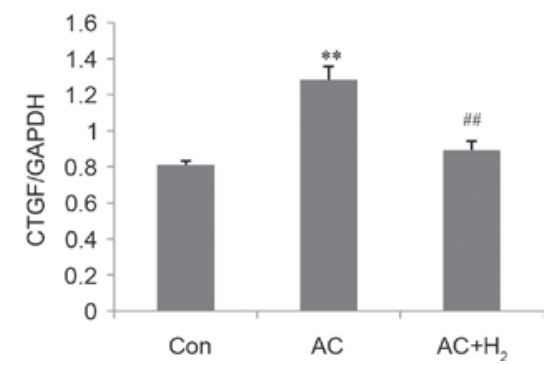

Figure 2. Effect of $\mathrm{H}_{2}$ on TGF- $\beta 1$ and its downstream signals in pressure-overloaded hearts. (A) TGF- $\beta 1$, p-Smad2/3, Smad2/3 and CTGF protein levels, as determined by western blotting; GAPDH was used as the loading control. Quantitative analysis of protein levels of (B) TGF- $\beta 1$, (C) the ratio of $\mathrm{p}-\mathrm{Smad} 2 / 3 / \mathrm{Smad} 2 / 3$ and (D) the protein levels of CTGF. Data are presented as the mean \pm standard deviation $(\mathrm{n}=3)$. ${ }^{*} \mathrm{P}<0.01,{ }^{* *} \mathrm{P}<0.001 \mathrm{vs}$. Con group; ${ }^{\#} \mathrm{P}<0.05,{ }^{\# \prime} \mathrm{P}<0.001 \mathrm{vs}$. AC group. TGF- $\beta 1$, transforming growth factor $\beta 1$; p-, phosphorylated-; CTGF, connective tissue growth factor; Con, control; AC, aortic constriction.

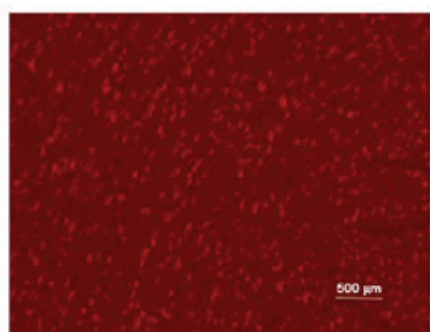

Con

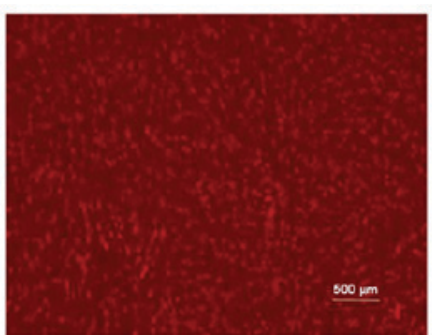

AC

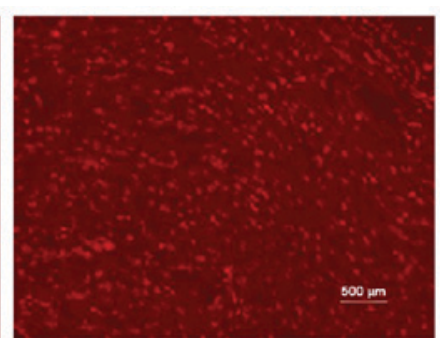

$\mathrm{AC}+\mathrm{H}_{2}$

Figure 3. Effect of $\mathrm{H}_{2}$ on reactive oxygen species levels in the left ventricle tissues. Representative image (magnification, x100) of 2,7-dichlorofluorescin diacetate staining in frozen sections by fluorescence microscopy. Con, control; AC, aortic constriction.

$\mathrm{H}_{2}$ restores $M D A$ levels and SOD activity in the myocardium. MDA levels and SOD activity are reliable indicators of oxidative stress and antioxidant capacity, respectively. AC resulted in a $65 \%$ increase in MDA content and a $43 \%$ decrease in SOD activity (all $\mathrm{P}<0.01$ ) compared with the Con group (Fig. 4). HCS treatment abrogated these effects, reducing MDA levels and increasing SOD activity (all $\mathrm{P}<0.05$ ) as compared with the AC group. These results indicate that hydrogen an antioxidant effect on the pressure-overloaded heart.

$\mathrm{H}_{2}$ inhibits NOX2 and NOX4 expression induced by pressure overload. The protein levels of NOX2 and NOX4 were illustrated in Fig. 5. Their expression of NOX2 and NOX4 were increased in the AC compared with the Con group (all $\mathrm{P}<0.001$ ). Subsequent to treatment with HCS, expression was significantly decreased compared with the AC group (all $\mathrm{P}<0.001)$. These results indicate that HCS treatment could attenuate oxidative stress by partially inhibiting the expression of NOX2 and NOX4.
$\mathrm{H}_{2}$ inhibits p38 MAPK activation induced by pressure overload. AC increased the percentage of phosphorylation of p38 MAPK by 3.6-fold as compared with control animals $(\mathrm{P}<0.001)$ (Fig. 6). HCS treatment decreased the ratio of p-p38 MAPK/p38 MAPK by $53 \%$ compared with the AC group $(\mathrm{P}<0.001)$.

\section{Discussion}

$\mathrm{H}_{2}$ has been widely used in clinical applications owing to its capacity to specifically neutralize cytotoxic oxygen radicals (24). Previously studies have demonstrated that $\mathrm{H}_{2}$ administration has protective effects against cerebral reperfusion injury, neurodegeneration and metabolic syndromes for patients by inhalation of $\mathrm{H}_{2}$ gas, intake of $\mathrm{H}_{2}$-dissolved water, or injection of $\mathrm{H}_{2}$-dissolved saline (25), among which injection of $\mathrm{H}_{2}$-dissolved saline ensures more accurate delivery of $\mathrm{H}_{2}$ (24). $\mathrm{H}_{2}$ has a protective effect on the cardiovascular system $(14,18,19,26-28)$; however, the role of $\mathrm{H}_{2}$ in pressure overload-induced cardiac fibrosis has not, to the best of our 

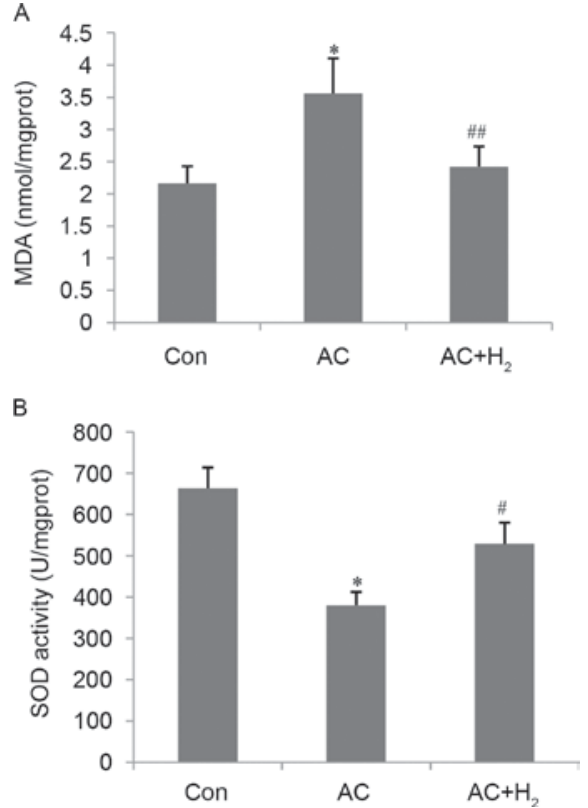

Figure 4. Myocardium MDA levels (A) and SOD activity (B) in the left ventricle. Data are presented as the mean \pm standard deviation $(n=4$ for $A$; $\mathrm{n}=3$ for $\mathrm{B}$ ). ${ }^{*} \mathrm{P}<0.01$ vs. control group; ${ }^{\#} \mathrm{P}<0.05,{ }^{\# \#} \mathrm{P}<0.01$ vs. AC group. MDA, malondialdehyde; SOD, superoxide dismutase; Con, control; AC, aortic constriction.

A

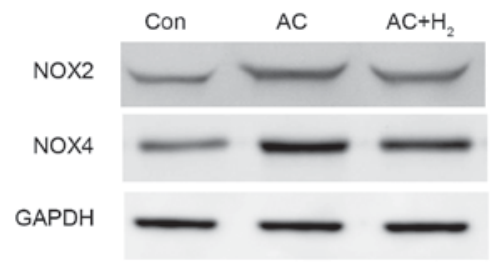

B

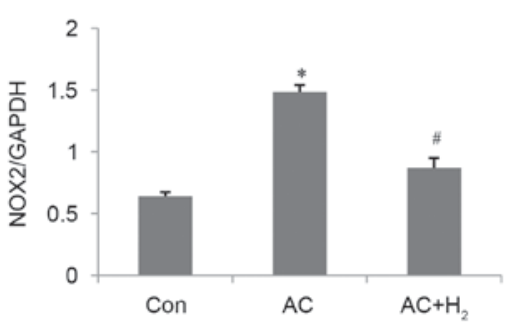

C

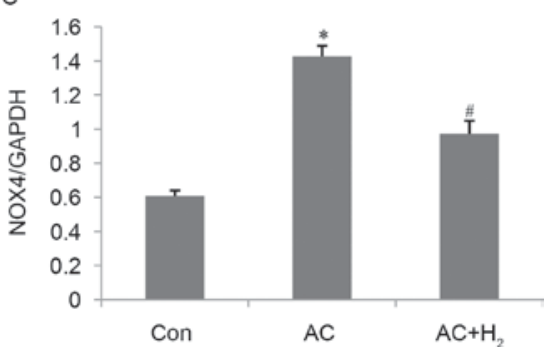

Figure 5. Effect of $\mathrm{H}_{2}$ on NOX2 and NOX4 protein expression in the left ventricle. GAPDH was used as a loading control. (A) NOX2 and NOX4 protein levels, as determined by western blotting. Quantitative analysis of (B) NOX2 and (C) NOX4 protein level. Data are presented as the mean \pm standard deviation $(n=3)$. ${ }^{*} \mathrm{P}<0.001$ vs. Con group; ${ }^{~} \mathrm{P}<0.001$ vs. AC group. NOX, nicotinamide adenine dinucleotide phosphate oxidases; Con, control; AC, aortic constriction.

knowledge, been previously investigated. The present study demonstrated that HCS treatment prevents interstitial fibrosis

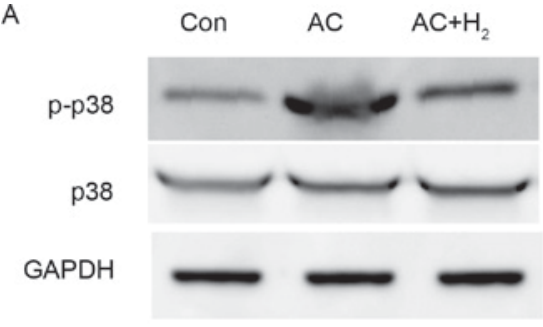

B

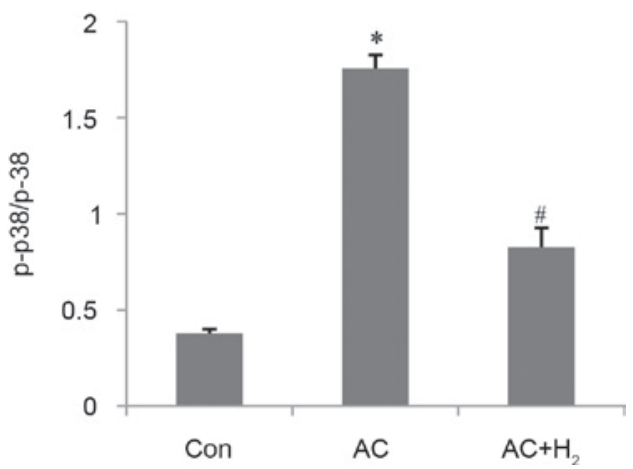

Figure 6. Effect of $\mathrm{H}_{2}$ on p-p38 MAPK and p38 MAPK protein expression in the left ventricle. GAPDH was used as the loading control. (A) p-p38 and p38 protein level, as determined by western blotting. (B) Quantitative analysis of the ratio of $\mathrm{p}-\mathrm{p} 38 / \mathrm{p} 38$. Data are presented as the mean \pm standard deviation $(n=3)$. * $\mathrm{P}<0.001$ vs. Con group; ${ }^{\text {P }}<0.001$ vs. AC group. p-, phosphorylated-; MAPK, mitogen-activated protein kinase; Con, control; AC, aortic constriction.

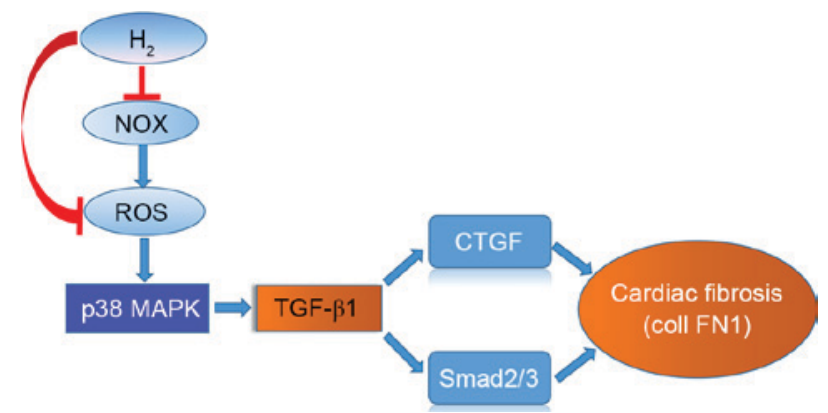

Figure 7. Schematic of the $\mathrm{H}_{2}$ mechanism. NOX, nicotinamide adenine dinucleotide phosphate oxidases; ROS, reactive oxygen species; MAPK, mitogen activated protein kinase; TGF, transforming growth factor; CTGF, connective tissue growth factor; Col I, collagen I; FN1, fibronectin 1.

and the progression of heart failure induced by pressure overload in rats. These effects were accompanied by changes in the levels of oxidative stress markers and the expression of pro-fibrotic factors. The results indicate that HCS may be effective for treating cardiac fibrosis and heart failure caused by hypertension-induced pressure overload.

Hypertension affects approximately 1 billion people worldwide and is a major public health challenge (29). Pressure overload induces early cardiac hypertrophy, fibrosis and diastolic dysfunction; when these persist, the heart becomes decompensated and dilated, eventually leading to systolic dysfunction (2). A previous study identified that fibrosis can promote the transition from left ventricular hypertrophy to heart failure (30). As such, reversing or preventing cardiac fibrosis is an important goal for improving the prognosis of 
patients with hypertensive heart disease. In the present study, cardiac fibrosis, LV dilation and systolic dysfunction were observed in rats in which pressure overload was induced by AC after 16 weeks. However, HCS treatment shortened LVDd and LVSd, reduced interstitial fibrotic area, and increased EF and FS, but did not decrease IVSd, LVPWd, LVW/BW or HW/BW ratios. The results indicated that initial cardiac hypertrophy progressed to cardiac fibrosis and heart failure (22), which were mitigated by $\mathrm{H}_{2}$. A previous study reported that HCS treatment attenuates left ventricular hypertrophy in spontaneously hypertensive rats, which differed from the results of the present study, potentially due to the fact that different time points were examined (19).

Oxidative stress serves a critical role in heart failure resulting from sustained pressure overload (31). The expression of oxidative stress markers is upregulated in the advent of heart failure (32), in addition to during the transition from hypertrophy to heart failure (33). It was demonstrated that the levels of ROS and MDA, an index of lipid peroxidation, were increased by $\mathrm{AC}$, an effect that was reversed by HCS treatment. It was previously observed that HCS treatment protected against doxorubicin-induced heart failure, which was accompanied by a downregulation of MDA levels (34). In addition, antioxidant capacity, as reflected by SOD, was impaired under pressure overload, and this was also ameliorated by HCS treatment.

ROS levels are increased in various types of heart diseases, including cardiac hypertrophy, fibrosis and heart failure $(35,36)$. ROS have been reported to activate TGF- $\beta 1$ to enhance ECM deposition in the interstitium (37). TGF- $\beta 1$ is an important fibrogenic factor that mediates the proliferation of fibroblast and conversion to myofibroblasts in addition to the production of ECM components including fibrillar collagen and fibronectin (2). CTGF is induced by TGF- $\beta 1$ through signaling cascades such as Smad2/3, and is essential for TGF- $\beta 1$-induced collagen synthesis (5). In the present study, Col I and FN1 mRNA levels and hydroxyproline content were upregulated under pressure overload. CTGF protein expression and the ratio of $\mathrm{p}-\mathrm{Smad} 2 / 3$ to $\mathrm{Smad} 2 / 3$ were additionally upregulated following TGF- $\beta 1$. Administration of HCS abolished these effects and reduced ECM deposition. These results are consistent with that of a previous study demonstrating that TGF- $\beta 1$ expression was induced by pressure overload, and that blocking its activity prevented myocardial fibrosis and cardiac dysfunction (38).

NOX are the main sources of ROS in the cardiovascular system, and among them, NOX2 and NOX4 serve an important role in the development of cardiac hypertrophy and interstitial fibrosis. p38 MAPK serves a key role in intracellular signal transduction in cardiac development and pathology (39). For example, p38 MAPK mediates the upregulation of TGF- $\beta 1$ induced by AngII, which is also dependent on NOX-induced ROS (7). In addition, ROS/p38 MAPK/TGF- $\beta 1$ signaling is essential for cardiac fibroblast proliferation (8). It was observed that pressure overload increased oxidative stress, p38 MAPK phosphorylation and TGF- $\beta 1$ expression; these were abrogated by HCS treatment, suggesting that $\mathrm{H}_{2}$ can inhibit ROS/p38 MAPK/TGF- $\beta 1$ signaling to improve interstitial fibrosis. However, intracellular ROS production should be directly measured and the effect of inhibiting NOX activity on TGF- $\beta 1$ expression must be analyzed in a further study.
$\mathrm{H}_{2}$ has been previously reported to suppress the production of cytotoxic oxygen radicals, including $\bullet \mathrm{OH}$ and $\mathrm{ONOO}^{-}(9)$; it also regulates gene expression and the phosphorylation of signaling proteins. $\mathrm{H}_{2}$ was demonstrated to inhibit the expression of matrix metalloproteinase (MMP)-2 and -9 (40) in addition to the phosphorylation of p38MAPK, exerting anti-allergenic and anti-inflammatory effects $(12,41)$. However, the primary molecular target of $\mathrm{H}_{2}$ remains unclear, and further studies are required to clarify the mechanism by which $\mathrm{H}_{2}$ regulates TGF- $\beta 1$ expression and to determine whether it directly attenuates cardiac fibrosis by negatively regulating MMP2 and -9 levels.

In conclusion, HCS treatment reduced interstitial fibrosis and improved cardiac function in pressure-overloaded rats, which was likely mediated by its anti-oxidative properties and via inhibition of p38 MAPK phosphorylation and TGF- $\beta 1$ downstream signaling. A schematic of the action of $\mathrm{H}_{2}$ is presented in Fig. 7. These data may provide a potential therapeutic application of $\mathrm{H}_{2}$ in preventing cardiac fibrosis and the progression of heart failure.

\section{Acknowledgements}

The authors would like to thank International Science Editing for the English language editing of the manuscript.

\section{References}

1. Braunwald E: The war against heart failure: The Lancet lecture. Lancet 385: 812-824, 2015.

2. Kong P, Christia P and Frangogiannis NG: The pathogenesis of cardiac fibrosis. Cell Mol Life Sci 71: 549-574, 2014.

3. Dobaczewski M, Chen W and Frangogiannis NG: Transforming growth factor (TGF) $-\beta$ signaling in cardiac remodeling. J Mol Cell Cardiol 51: 600-606, 2011.

4. Lim $\mathrm{H}$ and Zhu YZ: Role of transforming growth factor-beta in the progression of heart failure. Cell Mol Life Sci 63: 2584-2596, 2006.

5. Creemers EE and Pinto YM: Molecular mechanisms that control interstitial fibrosis in the pressure-overloaded heart. Cardiovasc Res 89: 265-272, 2011.

6. Murdoch CE, Zhang M, Cave AC and Shah AM: NADPH oxidase-dependent redox signalling in cardiac hypertrophy, remodelling and failure. Cardiovasc Res 71: 208-215, 2006.

7. Rosenkranz S: TGF-beta1 and angiotensin networking in cardiac remodeling. Cardiovasc Res 63: 423-432, 2004.

8. Yu M, Zheng Y, Sun HX and Yu DJ: Inhibitory effects of enalaprilat on rat cardiac fibroblast proliferation via ROS/P38MAPK/TGF- $\beta 1$ signaling pathway. Molecules 17: 2738-2751, 2012.

9. Ohsawa I, Ishikawa M, Takahashi K, Watanabe M, Nishimaki K, Yamagata K, Katsura K, Katayama Y, Asoh S and Ohta S: Hydrogen acts as a therapeutic antioxidant by selectively reducing cytotoxic oxygen radicals. Nat Med 13: 688-694, 2007.

10. Xie K, Yu Y, Pei Y, Hou L, Chen S, Xiong L and Wang G: Protective effects of hydrogen gas on murine polymicrobial sepsis via reducing oxidative stress and HMGB1 release. Shock 34: 90-97, 2010.

11. Cai J, Kang Z, Liu WW, Luo X, Qiang S, Zhang JH, Ohta S, Sun $\mathrm{X}, \mathrm{Xu} \mathrm{W}$, Tao $\mathrm{H}$ and Li R: Hydrogen therapy reduces apoptosis in neonatal hypoxia-ischemia rat model. Neurosci Lett 441: 167-172, 2008

12. Itoh T, Fujita Y, Ito M, Masuda A, Ohno K, Ichihara M, Kojima T, Nozawa Y and Ito M: Molecular hydrogen suppresses FcepsilonRI-mediated signal transduction and prevents degranulation of mast cells. Biochem Biophys Res Commun 389: 651-656, 2009.

13. Kamimura N, Nishimaki K, Ohsawa I and Ohta S: Molecular hydrogen improves obesity and diabetes by inducing hepatic FGF21 and stimulating energy metabolism in $\mathrm{db} / \mathrm{db}$ mice. Obesity (Silver Spring) 19: 1396-1403, 2011. 
14. Yoshida A, Asanuma H, Sasaki H, Sanada S, Yamazaki S, Asano Y, Shinozaki Y, Mori H, Shimouchi A, Sano M, et al: $\mathrm{H}(2)$ mediates cardioprotection via involvements of K(ATP) channels and permeability transition pores of mitochondria in dogs. Cardiovasc Drugs Ther 26: 217-226, 2012.

15. Sun Q, Kang Z, Cai J, Liu W, Liu Y, Zhang JH, Denoble PJ, Tao $\mathrm{H}$ and Sun X: Hydrogen-rich saline protects myocardium against ischemia/reperfusion injury in rats. Exp Biol Med (Maywood) 234: 1212-1219, 2009.

16. Ohsawa I, Nishimaki K, Yamagata K, Ishikawa M and Ohta S: Consumption of hydrogen water prevents atherosclerosis in apolipoprotein E knockout mice. Biochem Biophys Res Commun 377: 1195-1198, 2008.

17. Qian L, Cao F, Cui J, Wang Y, Huang Y, Chuai Y, Zaho L, Jiang H and Cai J: The potential cardioprotective effects of hydrogen in irradiated mice. J Radiat Res 51: 741-747, 2010.

18. Noda K, Shigemura N, Tanaka Y, Kawamura T, Hyun Lim S, Kokubo K, Billiar TR, Bermudez CA, Kobayashi H and Nakao A: A novel method of preserving cardiac grafts using a hydrogen-rich water bath. J Hear Lung Transplant 32: 241-250, 2013.

19. Yu YS and Zheng H: Chronic hydrogen-rich saline treatment reduces oxidative stress and attenuates left ventricular hypertrophy in spontaneous hypertensive rats. Mol Cell Biochem 365: 233-242, 2012

20. Regulations of People's Republic of China for Administration of Laboratory Animals. State Science and Technology Commission, Beijing, 1988.

21. Oharazawa H, Igarashi T, Yokota T, Fujii H, Suzuki H, Machide M, Takahashi H, Ohta S and Ohsawa I: Protection of the retina by rapid diffusion of hydrogen: Administration of hydrogen-loaded eye drops in retinal ischemia-reperfusion injury. Investig Ophthalmol Vis Sci 51: 487-492, 2010.

22. Ma Y, Chen Y, Yang Y, Chen B, Liu D, Xiong Z, Zhang C and Dong Y: Proteasome inhibition attenuates heart failure during the late stages of pressure overload through alterations in collagen expression. Biochem Pharmacol 85: 223-233, 2013.

23. Livak KJ and Schmittgen TD: Analysis of relative gene expression data using real-time quantitative PCR and the 2(-Delta Delta C(T)) method. Methods 25: 402-408, 2001.

24. Ohta S: Recent progress toward hydrogen medicine: Potential of molecular hydrogen for preventive and therapeutic applications. Curr Pharm Des 17: 2241-2252, 2011.

25. Ohta S: Molecular hydrogen as a preventive and therapeutic medical gas: Initiation, development and potential of hydrogen medicine. Pharmacol Ther 144: 1-11, 2014.

26. Hayashida K, Sano M, Ohsawa I, Shinmura K, Tamaki K, Kimura K, Endo J, Katayama T, Kawamura A, Kohsaka S, et al: Inhalation of hydrogen gas reduces infarct size in the rat model of myocardial ischemia-reperfusion injury. Biochem Biophys Res Commun 373: 30-35, 2008.
27. Hayashida K, Sano M, Kamimura N, Yokota T, Suzuki M, Maekawa Y, Kawamura A, Abe T, Ohta S, Fukuda K and Hori S: $\mathrm{H}(2)$ gas improves functional outcome after cardiac arrest to an extent comparable to therapeutic hypothermia in a rat model. J Am Heart Assoc 1: e003459, 2012.

28. Hayashi T, Yoshioka T, Hasegawa K, Miyamura M, Mori T, Ukimura A, Matsumura Y and Ishizaka N: Inhalation of hydrogen gas attenuates left ventricular remodeling induced by intermittent hypoxia in mice. Am J Physiol Heart Circ Physiol 301: H1062-H1069, 2011.

29. Kearney PM, Whelton M, Reynolds K, Muntner P, Whelton PK and He J: Global burden of hypertension: Analysis of worldwide data. Lancet 365: 217-223, 2005.

30. Lazzeroni D, Rimoldi O and Camici PG: From left ventricular hypertrophy to dysfunction and failure. Circ J 80: 555-564, 2016.

31. Sawyer DB, Siwik DA, Xiao L, Pimentel DR, Singh K and Colucci WS: Role of oxidative stress in myocardial hypertrophy and failure. J Mol Cell Cardiol 34: 379-388, 2002.

32. Van Kimmenade RR and Januzzi JL Jr: Emerging biomarkers in heart failure. Clin Chem 58: 127-138, 2012.

33. Dhalla AK, Hill MF and Singal PK: Role of oxidative stress in transition of hypertrophy to heart failure. J Am Coll Cardiol 28: 506-514, 1996.

34. Wu S, Zhu L, Yang J, Fan Z, Dong Y, Luan R, Cai J and Fu L: Hydrogen-containing saline attenuates doxorubicin-induced heart failure in rats. Pharmazie 69: 633-636, 2014.

35. Takimoto E and Kass DA: Role of oxidative stress in cardiac hypertrophy and remodeling. Hypertension 49: 241-248, 2007.

36. Tsutsui H, Kinugawa S and Matsushima S: Oxidative stress and heart failure. Am J Physiol Heart Circ Physiol 301: H2181-H2190, 2011.

37. Barcellos-Hoff MH and Dix TA: Redox-mediated activation of latent transforming growth factor-beta 1. Mol Endocrinol 10: 1077-1083, 1996

38. Kuwahara F, Kai H, Tokuda K, Kai M, Takeshita A, Egashira K and Imaizumi T: Transforming growth factor-beta function blocking prevents myocardial fibrosis and diastolic dysfunction in pressure-overloaded rats. Circulation 106: 130-135, 2002.

39. Wang Y: Mitogen-activated protein kinases in heart development and diseases. Circulation 116: 1413-1423, 2007.

40. Chen CH, Manaenko A, Zhan Y, Liu WW, Ostrowki RP, Tang $\mathrm{J}$ and Zhang JH: Hydrogen gas reduced acute hyperglycemia-enhanced hemorrhagic transformation in a focal ischemia rat model. Neuroscience 169: 402-414, 2010.

41. Cardinal JS, Zhan J, Wang Y, Sugimoto R, Tsung A, McCurry KR, Billiar TR and Nakao A: Oral hydrogen water prevents chronic allograft nephropathy in rats. Kidney Int 77: 101-109, 2010. 